\title{
DEA-BASED COMPETITION STRATEGY IN THE PRESENCE OF UNDESIRABLE PRODUCTS. AN APPLICATION TO PAPER MILLS
}

\author{
ALIREZA AMIRTEIMOORI ${ }^{1 *}$, SIMIN MASROURI $^{2}$ \\ ${ }^{1}$ Department of Applied Mathematics, Rasht Branch, Islamic Azad University, Rasht, Iran \\ ${ }^{2}$ Department of Mathematics, Lahijan Branch, Islamic Azad University, Lahijan, Iran
}

\begin{abstract}
In real applications of data envelopment analysis (DEA), there are cases in which undesirable outputs are produced along with desirable outputs in such a way that the total sum of the produced undesirable outputs over the production units must be fixed and constant. In this case, a trade-off between the decision-making units $(D M U s)$ is needed to balance the production of undesirable outputs. In a rational sight, this trade-off is done in such a way that all $D M U$ s improve their relative performances. In this paper, a single DEA-based model is proposed to model fixed and variable-sum undesirable outputs in production processes. A common equilibrium efficient frontier is constructed and after reallocating the input/output factors, all decision-making units (DMUs) prevail as efficient. A real case of 32 paper mills in China is given. The results of the analysis demonstrated that some economically developed paper mills have better performance than less developed paper mills; in particular, all efficient paper mills are the developed ones.
\end{abstract}

Keywords: data envelopment analysis, equilibrium efficient frontier, undesirable outputs, efficiency

\section{Introduction}

Governance in different countries has taken measures to solve environmental problems like the pollutant which is caused by industrial productions. In this sense, modelling undesirable products in the analysis of the performance of production processes takes on strategic importance in guiding the decisions of the decision-makers to reduce this undesirable factor and, hence, to reduce environmental impacts on climate and air quality. So, researchers proposed a lot of techniques to deal with undesirable outputs for measuring environmental efficiency. In this regard, one of the most frequently used and well-known decision-making tools is Data Envelopment Analysis (DEA),

*Corresponding author, email address: ateimoori@iaurasht.ac.ir Received 22 September 2020, accepted 7 May 2021 
which was initially introduced by Charnes, Cooper and Rhodes [4] and extended by Banker et al. [3].

DEA is a mathematical programming-based technique to evaluate the relative efficiency of a set of homogeneous decision-making units (DMUs) with multiple incommensurate inputs and outputs. Classic DEA models assume that there are two types of factors affecting the performance of the DMUs: input variables, and output variables, and DEA attempts to evaluate the $D M U$ s which is a $D M U$ 's success in producing more outputs by consuming fewer inputs. In real applications, however, undesirable outputs or other harmful ones are produced along with the desired outputs and modelling undesirable outputs using tools, such as DEA or other nonparametric techniques are important and have attracted considerable attention among researchers.

Many DEA-based attempts have been made to model undesirable outputs. Hailu and Veeman [7] use a classic DEA model to evaluate the relative efficiency of the DMUs in the presence of desirable and undesirable outputs. In their model, undesirable outputs are considered as inputs. Seiford and Zho [14] resort to data translation to model undesirable outputs and inputs in classic DEA models. By reversing undesirable outputs, they reduce bad outputs in traditional DEA models. Färe and Grosskopf [5] claim that considering undesirable outputs as inputs is not consistent with physical laws and they use the weak disposability assumption of Shepherd [15] to define their weakly disposable technology. They apply a single abatement factor to all of the firms to allow for the simultaneous reduction of desirable and undesirable outputs.

Instead of a single abatement factor, Kuosmanen [9] employed non-uniform abatement factors to the $D M U$ s and he claimed that the correct and complete technology set is the one that he proposed. Khoshandam et al. [8] present an approach to determine marginal rates or elasticities of substitution of the production units. They use a DEA-based procedure to calculate group marginal rates of substitution and to this end, the envelopment form of the BCC model is directly applied to analyse the trade-offs and marginal rates of substitution. Amiteimoori et al. [1] offer an alternative definition of weak disposability in an additive form. Their new definition applies a fixed reduction factor to each input/output measure. This reduction factor decreases the level of undesirable factors by decreasing the activity level of the DMUs. Pham and Zelenyuk [12] shed some light on the debate of handling undesirable outputs from various theoretical and practical viewpoints, including disposability, convexity, returns to scale, and computational issues. They also propose an alternative model and unveiled some interesting properties of the model to construct a comprehensive taxonomy of reference technology sets for activity analysis models in variable returns to scale environment. Zare-Haghighi et al. [21] calculate the congestion of inputs in the presence of desirable and undesirable outputs. Regarding undesirable outputs, Sadri et al. [13] suggest an alternative method for optimisation of inefficient cost units in the presence of undesirable products.

In all these studies on undesirable factors, desirable and undesirable outputs are assumed to be variable-sum. In other words, there is no need that the summation of the 
variables to be constant. In many real applications, however, we confront such cases in which the total sum of some undesirable output is constant and we just can make a trade-off between the $D M U$ s to make all $D M U$ s to be efficient. Consider, for example, the production process in paper mills that produce paper along with environmental pollutions such as biochemical oxygen demand. These pollutions, caused by paper mills, are poured into the rivers. The standard capacity of the rivers is limited and we cannot pour unlimited pollutions into the rivers. In this sense, if a mill increases, its pollutant production quota, to maintain balance, another mill must reduce its quota. So, we can just make a trade-off between the mills and the total amount of pollutions must be a fixed constant.

In the last decade, a lot of studies have been done in the field of DEA with fixed-sum inputs and outputs. Lins et al. [10] propose a zero-sum gain DEA model to measure the efficiency of the countries that participated in the Olympic Games. Gomes and Lins [6] introduce the zero-sum gain DEA model, where the sum of the quantities produced by all $D M U$ s can be set as the upper admissible bound. Yang et al. [18] propose a fixed-sum desirable output DEA approach based on a minimal reduction competition strategy that incorporates free competition. This approach is developed by Yang et al. [19] and they show that any inefficient $D M U$ in a competition takes a quantity from efficient $D M U$ s and achieve a common equilibrium efficient frontier. In this method, the inefficient $D M U$ s achieve the efficient frontier step by step, and it depends on the order of selecting inefficient $D M U$ s to achieve an equilibrium efficient frontier. So, a different starting $D M U$ leads to a different result. To overcome this, Yang et al. [20] develop a onestep algorithm based on the extended minimal adjustment strategy and the equilibrium competition strategy to achieve a common equilibrium efficient frontier. Wu et al. [17] give a fixed-sum output DEA model to show competition to $D M U$ s with fixed-sum undesirable outputs. Amirteimoori et al. [2] suggest a context-based competition strategy to analyse the performance of bank branches with fixed-sum outputs. Their approach uses context-dependent DEA that refers to a DEA approach where a set of DMUs is assessed against a particular assessment context. Zhang et al. [22] study two DEA-based resource allocation models and apply their model to measure the performances of 30 regions in China. Momeni et al. [11] develop a centralised DEA model to reallocate emission permits in the Cap and trade system based on countries efficiencies. Their model considers all $D M U$ s together and improves the whole efficiency of the $D M U$ s by reducing total emission permit as undesirable outputs.

The purpose of this study is to make a trade-off between the fixed-sum factors of the $D M U$ s to make all firms as efficient. Toward this end, a common equilibrium efficient frontier is constructed and in this process, the linearity of the model is preserved. We assume that the desirable and undesirable outputs are interdependent and to reduce one of these two factors, the other one must also be reduced. In this case, we will use the weak disposability assumption of Shephard [15] to handle these desirable and undesirable outputs in the process. A real case on 32 paper mills in the Anhui province and the Huai River of China is given. 
The rest of the paper is organised as follows: In Section 2, we review the approach proposed by $\mathrm{Wu}$ et al. [17]. In Section 3, the proposed approach to construct a common equilibrium efficient frontier is given. A real case on 32 China's paper mills taken from $\mathrm{Wu}$ et al. [16] is given in Section 4. Conclusions are presented in Section 5.

\section{Preliminaries}

In this section, we first review the prior works that evaluate the relative performances of the $D M U$ s with fixed-sum undesirable outputs. Suppose there are $N D M U$ s, each unit produces $S$ variant sum desirable outputs $y_{r j}: r=1, \ldots, S, j=1, \ldots, N$ and $T$ fixed-sum undesirable outputs $f_{t j}: t=1, \ldots, T, j=1, \ldots, N$ using $M$ inputs $x_{i j}: i=1, \ldots, M, j=1, \ldots, N$. The total variable outputs can be increased while the summation over undesirable outputs is constant. $\mathrm{Wu}$ et al. [17] proposed a fixed-sum output DEA model to show competition over $D M U \mathrm{~s}$ with fixed-sum undesirable outputs. They stated that a $D M U$ is efficient if undesirable outputs are reduced as much as possible. For this purpose, a $D M U$ must reduce its undesirable outputs, and this reduction should be offset by other $D M U$ s since the summation of undesired outputs is constant. They proposed the following model:

$$
\max \frac{\sum_{r=1}^{S} u_{r}\left(y_{r k}+\alpha_{r}\right)+u_{o}}{\sum_{i=1}^{M} v_{i} x_{i k}+\sum_{t=1}^{T} w_{t}\left(f_{t k}-\beta_{t}\right)}
$$

s.t.

$$
\begin{gathered}
\frac{\sum_{r=1}^{S} u_{r}\left(y_{r k}+\alpha_{r}\right)+u_{o}}{\sum_{i=1}^{M} v_{i} x_{i k}+\sum_{t=1}^{T} w_{t}\left(f_{t k}-\beta_{t}\right)} \leq 1 \\
\frac{\sum_{r=1}^{S} u_{r} y_{r j}+u_{o}}{\sum_{i=1}^{M} v_{i} x_{i j}+\sum_{t=1}^{T}\left(f_{t j}+s_{t j}\right)} \leq 1, j \neq k \\
\beta_{t}=\sum_{\substack{j=1 \\
j \neq k}}^{N} s_{t j}, 0 \leq \beta_{t} \leq f_{t k}, t=1, \ldots, T \\
v_{i}, u_{r}, w_{t}, \alpha_{r} \geq 0 \text { for all } i, r, t
\end{gathered}
$$


Model (1) implies that the improved $D M U_{k}:\left(x_{k}, f_{k}-\beta, y_{k}+\alpha\right)$ can certainly reach the production frontier. This model has two steps. At the first step, the weighted sum of output changes are minimised, and in this sense $D M U_{k}$ is improved. To this end, we use the following program:

$$
\min \sum_{r=1}^{S} u_{r} \alpha_{r}+\sum_{t=1}^{T} w_{t} \beta_{t}
$$

s.t.

$$
\begin{aligned}
& \frac{\sum_{r=1}^{S} u_{r}\left(y_{r k}+\alpha_{r}\right)+u_{0}}{\sum_{i=1}^{M} v_{i} x_{i k}+\sum_{t=1}^{T} w_{t}\left(f_{t k}-\beta_{t}\right)}=1 \\
& \frac{\sum_{r=1}^{S} u_{r}\left(y_{r j}+\alpha_{r}\right)+u_{0}}{\sum_{i=1}^{M} v_{i} x_{i j}+\sum_{t=1}^{T} w_{t}\left(f_{t j}+s_{t j}\right)} \leq 1, j \neq k \\
& \beta_{t}=\sum_{\substack{j=1 \\
j \neq k}}^{N} s_{t j}, 0 \leq \beta_{t} \leq f_{t k}, t=1, \ldots, T \\
& v_{i}, u_{r}, w_{t}, \alpha_{r} \geq 0 \text { for all } i, r, t
\end{aligned}
$$

At the second step, each $D M U_{k}$ (as a $D M U$ under evaluation) is evaluated to show the improvement in relative efficiency. They measure the performance of each adjusted $D M U$ by solving the following program:

$$
E_{k}=\max \frac{\sum_{r=1}^{S} u_{r} y_{r k}+u_{0}}{\sum_{i=1}^{M} v_{i} x_{i k}+\sum_{t=1}^{T} w_{t} f_{t k}}
$$

s.t.

$$
\frac{\sum_{r=1}^{S} u_{r}\left(y_{r k}+\hat{\alpha}_{r}\right)+u_{0}}{\sum_{i=1}^{M} v_{i} x_{i k}+\sum_{t=1}^{T} w_{t}\left(f_{t k}-\hat{\beta}_{t}\right)} \leq 1
$$




$$
\begin{gathered}
\frac{\sum_{r=1}^{S} u_{r} y_{r j}+u_{0}}{\sum_{i=1}^{M} v_{i} x_{i j}+\sum_{t=1}^{T} w_{t}\left(f_{t j}+\widehat{s}_{t j}\right)} \leq 1, j \neq k \\
\beta_{t}=\sum_{\substack{j=1 \\
j \neq k}}^{N} s_{t j}, 0 \leq \beta_{t} \leq f_{t k}, t=1, \ldots, T \\
v_{i}, u_{r}, w_{t} \geq 0 \text { for all } i, r, t
\end{gathered}
$$

in which $\widehat{\alpha}_{r}, \widehat{\beta}_{t}, \widehat{s}_{t j}$ is an optimal solution of model (2). In the approach proposed by Wu et al. [17], undesirable outputs are considered as inputs. Although Färe and Grosskopf [5] claim that this may be inconsistent with physical laws, the authors of this paper think that this may be correct if the desirable and undesirable outputs are not interdependent. In other words, when desirable outputs and undesirable outputs are independent, considering undesirable outputs as inputs may be correct. However, if these two outputs are directly correlated, a weak disposability assumption may be useful in handling undesirable outputs (such as producing electricity and emissions of harmful in power plants).

In the next section, we will use the weakly disposable technology of Kuosmanen [9] to model undesirable outputs as outputs.

\section{The proposed approach}

As we stated in the previous section, modelling undesirable outputs as inputs is not consistent with physical laws and it cannot reflect a good estimation of efficiency. So, in the proposed model, we will use the weak disposability assumption of Shephard [15] to model undesirable outputs as outputs. Since we want to reduce the inputs and increase the desirable outputs and we want to make a reallocation of the fixed-sum undesirable outputs, we will use the directional distance function in the weakly disposable technology set of Kuosmanen [9].

Obviously, the sum of variable-sum inputs and outputs can be decreased and increased, respectively. Also, in fixed-sum undesirable outputs we must have $\sum_{j=1}^{N} f_{t j}=F_{t}: t=1, \ldots, T$ in which $F_{t}$ is a constant.

Consider the directional model of Kuosmanen [9] that uses the weakly disposable technology set as underlying technology set: 


$$
\begin{aligned}
& \rho_{o}^{*}=\max \rho_{o} \\
& \text { s.t. } \\
& \sum_{j=1}^{N}\left(\lambda_{j}+\mu_{j}\right) x_{i j} \leq x_{i o}-\rho_{o} d_{i}^{x}, i=1, \ldots, M \\
& \sum_{j=1}^{N} \lambda_{j} y_{r j} \geq y_{r o}+\rho_{o} d_{r}^{y}, r=1, \ldots, \mathrm{S} \\
& \sum_{j=1}^{N} \lambda_{j} f_{t j}=f_{t o}-\rho_{o} d_{t}^{f}, t, \ldots, T \\
& \sum_{j=1}^{N}\left(\lambda_{j}+\mu_{j}\right)=1 \\
& \lambda_{j}, \mu_{j} \geq 0, j=1, \ldots, N
\end{aligned}
$$

in which the non-zero vector $\left(d^{x}, d^{y}, d^{f}\right) \geq 0$ is a user-defined direction and it shows the direction for the improvements of the inputs, desirable outputs and undesirable outputs, respectively. In model (4), the desirable outputs are increased, undesirable outputs and inputs are decreased in a user-defined direction $\left(d^{x}, d^{y}, d^{f}\right)$. Clearly, $\rho_{o}^{*} \geq 0$ and $D M U_{o}$ is efficient if and only if $\rho_{o}^{*}=0$.

In our proposed fixed-sum outputs reallocation model, we will use the multiplier model. So, we need to provide the dual formulation of model (4). The dual formulation of model (4) is as follows:

$$
\begin{aligned}
& \min \sum_{i=1}^{M} v_{i} x_{i o}-\sum_{r=1}^{S} u_{r} y_{r o}+\sum_{t=1}^{T} w_{t} f_{t o}+\varphi \\
& \text { s.t. } \\
& \sum_{i=1}^{M} v_{i} x_{i j}-\sum_{r=1}^{S} u_{r} y_{r j}+\sum_{t=1}^{T} w_{t} f_{t j}+\varphi \geq 0, j=1, \ldots, N \\
& \sum_{i=1}^{M} v_{i} x_{i j}+\varphi \geq 0, \quad j=1, \ldots, N \\
& \sum_{i=1}^{M} v_{i} d_{i}^{x}-\sum_{r=1}^{S} u_{r} d_{r}^{y}+\sum_{t=1}^{T} w_{t} d_{t}^{f}=1 \\
& v_{i}, u_{r} \geq 0 \text { for all } i, r
\end{aligned}
$$

In the dual formulation, $v_{i}, u_{r}$ and $w_{t}$ are the dual variables associated with the inputs, good outputs, and undesirable outputs constraints, respectively. The variable $\varphi$ is associated with the convexity constraint of the primal problem. 
Now, we use the dual formulation (5) to obtain an equilibrium efficient frontier. In the model we will propose, the inputs are reduced, the variable-sum desirable outputs are increased, and the fixed-sum undesirable outputs are changed in such a way that all $D M U$ s are projected onto the equilibrium efficient frontier. To construct an equilibrium efficient frontier, the following mathematical programming problem is proposed:

$$
\begin{aligned}
& \min \sum_{j=1}^{N} \sum_{t=1}^{T} w_{t}\left|\delta_{t j}\right|+\sum_{j=1}^{N} \sum_{r=1}^{S} u_{r} \beta_{r j}+\sum_{j=1}^{N} \sum_{i=1}^{M} v_{i} \alpha_{i j} \\
& \text { s.t. } \\
& \sum_{i=1}^{M} v_{i}\left(x_{i j}-\alpha_{i j}\right)-\sum_{r=1}^{S} u_{r}\left(y_{r j}+\beta_{r j}\right)+\sum_{t=1}^{T} w_{t}\left(f_{t j}+\delta_{t j}\right)+\varphi=0, j=1, \ldots, N \\
& \sum_{i=1}^{M} v_{i}\left(x_{i j}-\alpha_{i j}\right)+\varphi \geq 0, j=1, \ldots, N \\
& \sum_{j=1}^{N} \delta_{t j}=0, t=1, \ldots, T \\
& f_{t j}+\delta_{t j} \geq 0 \text { for all } j, t \\
& x_{i j}-\alpha_{i j} \geq 0 \text { for all } j, i \\
& y_{r j}+\beta_{r j} \geq 0 \text { for all } j, r \\
& f_{t j}+\delta_{t j} \leq M_{t j} \text { for all } j, r \\
& v_{i}, u_{r}, \alpha_{i j}, \beta_{r j} \geq 0
\end{aligned}
$$

In model (6), $\alpha_{i j}$ is used to reduce the $i$ th input of $D M U_{j}, \beta_{r j}$ is used to increase the $r$ th desirable output of $D M U_{j}$, and $\delta_{t j}$ is used to change the $t$ th undesirable output of $D M U_{j}$. The first $N$ equality constraints guarantee that the trade-off is done in such a way that all new $D M U$ s are efficient. To preserve the balance, we impose the constraint $\sum_{j=1}^{N} \delta_{t j}=0, t=1, \ldots, T$. Because we are interested in making all $D M U$ s efficient with minimum changes, in the objective function, the weighted sum of these adjustment variables are minimised. Note that $\delta_{t j}$ is unrestricted in sign, because we may need to reduce from one $D M U$ and to increase to another $D M U$. In this sense, we minimise the weighted sum of its absolute value.

In the seventh constraint, $M_{t j}$ is a user-defined value to show the maximum value of the pollutant that is allowed to be poured to the rivers. Model (6) guarantees that after changing the inputs and outputs, all improved $D M U$ s can reach the production frontier. 
This frontier is called equilibrium efficient frontier that is a strong efficient frontier of the production possibility set.

Model (6) is a nonlinear model, but it can be transformed into a linear form in two steps. First, make the changes of variables $\alpha_{i j}^{\prime}=v_{i} \alpha_{i j}, \beta_{r j}^{\prime}=u_{r} \beta_{r j}, \delta_{t j}^{\prime}=w_{t} \delta_{t j}$. In this case, model (6) is transformed into the following form:

$$
\begin{aligned}
& \min \sum_{j=1}^{N} \sum_{t=1}^{T}\left|\delta_{t j}^{\prime}\right|+\sum_{j=1}^{N} \sum_{r=1}^{S} \beta_{r j}^{\prime}+\sum_{j=1}^{N} \sum_{i=1}^{M} \alpha_{i j}^{\prime} \\
& \text { s.t. } \\
& \sum_{i=1}^{M}\left(v_{i} x_{i j}-\alpha_{i j}^{\prime}\right)-\sum_{r=1}^{S}\left(u_{r} y_{r j}+\beta_{r j}^{\prime}\right)+\sum_{t=1}^{T}\left(w_{t} f_{t j}+\delta_{t j}^{\prime}\right)+\varphi=0, j=1, \ldots, N \\
& \sum_{i=1}^{S}\left(v_{i} x_{i j}-\alpha_{i j}^{\prime}\right)+\varphi \geq 0, j=1, \ldots, N \\
& \sum_{j=1}^{N} \delta_{t j}^{\prime}=0, t=1, \ldots, T \\
& w_{t} f_{t j}+\delta_{t j}^{\prime} \geq 0 \text { for all } t, j \\
& v_{i} x_{i j}-\alpha_{i j}^{\prime} \geq 0 \text { for all } i, j \\
& u_{r} y_{r j}+\beta_{r j}^{\prime} \geq 0 \text { for all } r, j \\
& w_{t} f_{t j}+\delta_{t j}^{\prime} \leq w_{t} M_{t j} \text { for all } t, j \\
& v_{i}, u_{r}, \alpha_{i j}^{\prime}, \beta_{r j}^{\prime} \geq 0
\end{aligned}
$$

Since the absolute values of $\delta_{t j}^{\prime}$ appear in the objective function, model (7) is not a linear programming problem. Now, suppose

$$
A_{t j}=\frac{1}{2}\left(\left|\delta_{t j}^{\prime}\right|+\delta_{t j}^{\prime}\right) \geq 0 \text { and } B_{t j}=\frac{1}{2}\left(\left|\delta_{t j}^{\prime}\right|-\delta_{t j}^{\prime}\right) \geq 0
$$

with $\left|\delta_{t j}^{\prime}\right|=A_{t j}+B_{t j}$ and $\delta_{t j}^{\prime}=A_{t j}-B_{t j}$. Using these changes of variables, model (7) can be written as follows:

$$
\begin{aligned}
& \min \sum_{j=1}^{N} \sum_{t=1}^{T}\left(A_{t j}+B_{t j}\right)+\sum_{j=1}^{N} \sum_{r=1}^{S} \beta_{r j}^{\prime} \gamma_{i j}^{\prime}+\sum_{j=1}^{N} \sum_{i=1}^{M} \alpha_{i j}^{\prime} \\
& \text { s.t. } \\
& \sum_{i=1}^{M}\left(v_{i} x_{i j}-\alpha_{i j}^{\prime}\right)-\sum_{r=1}^{S}\left(u_{r} y_{r j}+\beta_{r j}^{\prime}\right)+\sum_{t=1}^{T}\left(w_{t} f_{t j}+A_{t j}-B_{t j}\right)+\varphi=0, j=1, \ldots, N
\end{aligned}
$$




$$
\begin{aligned}
& \sum_{i=1}^{M}\left(v_{i} x_{i j}-\alpha_{i j}^{\prime}\right)+\varphi \geq 0, j=1, \ldots, N \\
& \sum_{j=1}^{N}\left(A_{t j}-B_{t j}\right)=0, t=1, \ldots, T \\
& w_{t} f_{t j}+A_{t j}-B_{t j} \geq 0, \text { for all } t, j \\
& v_{i} x_{i j}-\alpha_{i j}^{\prime} \geq 0, \text { for all } i, j \\
& w_{t} f_{t j}+A_{t j}-B_{t j} \leq w_{t} M_{t j}, \text { for all } t, j \\
& v_{i}, u_{r}, \alpha_{i j}^{\prime}, \beta_{r j}^{\prime}, A_{t j}, B_{t j} \geq 0, \text { for all } i, t, r, j
\end{aligned}
$$

Model (8) is a linear programming problem. It is easy to show that the origin is a feasible solution to model (8) and this guarantees the feasibility and boundedness of the model. By solving this model, a common equilibrium efficient frontier is constructed and based on the reallocation of the fixed-sum undesirable outputs, all DMUs are projected onto this frontier and, in this case, all $D M U$ s prevail as efficient.

In some real applications, there is an upper limit to undesirable outputs. We are interested in studying if we use this upper limit of undesirable output, how the efficiency and paper production would change, or if we gain anything substantial. To this end, we solve the following linear programming problem:

$\max \rho$

s.t.

$$
\begin{aligned}
& \sum_{j=1}^{N}\left(\lambda_{j}+\mu_{j}\right) x_{i j} \leq x_{i o}, i=1, \ldots, M \\
& \sum_{j=1}^{N} \lambda_{j} y_{r j} \geq \rho y_{r o}, r=1, \ldots, S \\
& \sum_{j=1}^{N} \lambda_{j} f_{t j}+s_{t j}=f_{t o}^{\max }, t, \ldots, T \\
& \sum_{j=1}^{N}\left(\lambda_{j}+\mu_{j}\right)=1, j=1, \ldots, N \\
& \lambda_{j}, \mu_{j} \geq 0, j=1, \ldots, N
\end{aligned}
$$

in which $f_{t o}^{\max }$ shows the maximum value of $t$ th undesirable-quota (the upper limit of undesirable) of $j$ th mill, taken from the 6th column of Table 1 . In this model, the fixed-sum assumption is ignored for undesirable outputs, and we are interested to know if we use the upper limit of undesirable quota output, how the efficiency of the firms would change. In other words, if we use the upper limit of undesirable-quota, do we gain anything substantial? To this end, we have solved model (9). 


\section{A real case on paper mills}

After formulating the methodological framework, we illustrate our proposed approach through analysis on a real data set on 32 paper mills along the Huai River in Anhui Province of China. This data set has been taken from Wu et al. [16]. Each paper mill uses two inputs of labor and capital to produce a single desirable output paper product. Along with the production of paper products, the bad output of biochemical oxygen demand (BOD) is also produced, which is a pollutant to the environment.

Table 1. The data set for the paper mills

\begin{tabular}{|c|c|c|c|c|c|}
\hline Mill & Labor & Capital & Paper & BOD & BOD-quota \\
\hline 1 & 1077 & 2.9599 & 2.7582 & 21.429 & 26.8452 \\
\hline 2 & 452 & 3.589 & 2.9514 & 19.8062 & 23.3606 \\
\hline 3 & 319 & 5.9019 & 1.47 & 12.3287 & 18.3147 \\
\hline 4 & 1075 & 4.8928 & 2.2354 & 9.1559 & 11.163 \\
\hline 5 & 813 & 4.0797 & 2.0669 & 11.9146 & 11.9146 \\
\hline 6 & 850 & 5.2396 & 0.8222 & 5.2037 & 10.0819 \\
\hline 7 & 1090 & 3.0228 & 1.5066 & 3.6054 & 9.0494 \\
\hline 8 & 122 & 3.1731 & 0.8066 & 3.7278 & 6.5775 \\
\hline 9 & 297 & 2.2774 & 1.9125 & 8.0765 & 13.8485 \\
\hline 10 & 1047 & 1.4919 & 0.7601 & 8.906 & 12.6009 \\
\hline 11 & 1010 & 3.9401 & 1.1579 & 4.894 & 10.5167 \\
\hline 12 & 262 & 3.2365 & 2.3216 & 4.0835 & 4.5835 \\
\hline 13 & 551 & 4.4486 & 2.1698 & 4.875 & 7.9018 \\
\hline 14 & 671 & 1.7897 & 0.8127 & 4.5334 & 7.0692 \\
\hline 15 & 577 & 2.3109 & 1.1549 & 6.1362 & 8.5654 \\
\hline 16 & 208 & 3.3982 & 1.0295 & 7.0186 & 12.386 \\
\hline 17 & 667 & 5.3319 & 2.9881 & 28.4877 & 33.2039 \\
\hline 18 & 878 & 3.4504 & 1.9076 & 13.168 & 14.168 \\
\hline 19 & 640 & 3.1098 & 1.2176 & 6.1616 & 10.6113 \\
\hline 20 & 927 & 3.3452 & 0.5187 & 1.4533 & 1.5042 \\
\hline 21 & 167 & 4.3297 & 2.4005 & 22.5809 & 27.3043 \\
\hline 22 & 903 & 3.8552 & 2.3085 & 26.339 & 29.1779 \\
\hline 23 & 720 & 1.9083 & 0.6545 & 3.0787 & 3.0787 \\
\hline 24 & 629 & 3.4682 & 2.7599 & 21.7332 & 24.5954 \\
\hline 25 & 152 & 5.5717 & 2.3748 & 5.3061 & 6.1214 \\
\hline 26 & 1010 & 4.6471 & 1.7323 & 9.136 & 10.2507 \\
\hline 27 & 578 & 2.5133 & 1.0617 & 5.0049 & 5.5956 \\
\hline 28 & 384 & 2.2474 & 0.9083 & 4.4373 & 8.1642 \\
\hline 29 & 166 & 3.9681 & 1.5151 & 10.6127 & 15.9031 \\
\hline 30 & 894 & 1.3685 & 0.9911 & 4.7758 & 6.5614 \\
\hline 31 & 143 & 5.3502 & 2.526 & 17.4677 & 22.0696 \\
\hline 32 & 879 & 2.8732 & 2.7721 & 26.9134 & 31.6652 \\
\hline Sum & 20158 & 113.0903 & 54.5731 & 342.3508 & 444.7538 \\
\hline & & & & & \\
\hline
\end{tabular}


The data set is shown in Table 1. A point to be noted is that to avoid contaminating the river, each paper mill has quota for polluting the river and maximum amount of pollution produced by each paper mill should not exceed the specified limit. The last column of Table 1 shows the upper limit for BOD.

Table 2. Projection point of model (4)

\begin{tabular}{|c|c|c|c|c|c|}
\hline Mill & Efficiency & Labor- $N$ & Capital- $N$ & Paper- $N$ & BOD- $N$ \\
\hline 1 & 0 & 1077 & 2.9599 & 2.7582 & 21.429 \\
\hline 2 & 0 & 452 & 3.589 & 2.9514 & 19.8062 \\
\hline 3 & 0.372 & 200.371 & 3.7071 & 2.0167 & 7.7439 \\
\hline 4 & 0.112 & 255.8775 & 4.3452 & 2.4856 & 8.1311 \\
\hline 5 & 0.206 & 432.9462 & 3.2407 & 2.492 & 9.4643 \\
\hline 6 & 0.516 & 411.6091 & 2.5373 & 1.2463 & 2.5199 \\
\hline 7 & 0.118 & 454.8873 & 2.6664 & 1.6842 & 3.1803 \\
\hline 8 & 0 & 122 & 3.1731 & 0.8066 & 3.7278 \\
\hline 9 & 0 & 297 & 2.2774 & 1.9125 & 8.0765 \\
\hline 10 & 0 & 1047 & 1.4919 & 0.7601 & 8.906 \\
\hline 11 & 0.357 & 500.1414 & 2.5326 & 1.5715 & 3.1458 \\
\hline 12 & 0 & 262 & 3.2365 & 2.3216 & 4.0835 \\
\hline 13 & 0.078 & 225.0819 & 4.0202 & 2.3395 & 4.4938 \\
\hline 14 & 0.029 & 651.5009 & 1.7377 & 1.0095 & 4.4017 \\
\hline 15 & 0.133 & 500.2962 & 2.0037 & 1.3084 & 5.3205 \\
\hline 16 & 0.133 & 180.3006 & 2.9457 & 1.242 & 6.0839 \\
\hline 17 & 0 & 667 & 5.3319 & 2.9881 & 28.4877 \\
\hline 18 & 0.196 & 506.5379 & 2.7744 & 2.2814 & 10.588 \\
\hline 19 & 0.257 & 475.7407 & 2.3117 & 1.5301 & 4.5802 \\
\hline 20 & 0.229 & 715.0241 & 1.8896 & 0.6373 & 1.121 \\
\hline 21 & 0 & 167 & 4.3297 & 2.4005 & 22.5809 \\
\hline 22 & 0.195 & 727.0678 & 3.1041 & 2.7583 & 21.2074 \\
\hline 23 & 0.083 & 660.5011 & 1.7506 & 0.7086 & 2.8243 \\
\hline 24 & 0.037 & 605.601 & 3.3392 & 2.8626 & 20.9247 \\
\hline 25 & 0 & 152 & 5.5717 & 2.3748 & 5.3061 \\
\hline 26 & 0.334 & 337.6866 & 3.0963 & 2.3104 & 6.0871 \\
\hline 27 & 0.152 & 489.8948 & 2.1302 & 1.2235 & 4.242 \\
\hline 28 & 0.036 & 370.1082 & 2.1661 & 0.9956 & 4.2768 \\
\hline 29 & 0.063 & 155.4833 & 3.7167 & 1.6111 & 9.9403 \\
\hline 30 & 0 & 894 & 1.3685 & 0.9911 & 4.7758 \\
\hline 31 & 0 & 143 & 5.3502 & 2.526 & 17.4677 \\
\hline 32 & 0 & 879 & 2.8732 & 2.7721 & 26.9134 \\
\hline Sum & & 15015.658 & 97.5685 & 49.8747 & 311.838 \\
\hline
\end{tabular}

To evaluate the relative efficiencies of the mills, model (4) is applied to this data set. The direction we have used in this example is $\left(d^{x}, d^{y}, d^{f}\right)=\left(x_{o}, y_{o}, f_{o}\right)$. The results are listed in the second column of Table 2.12 mills are relatively efficient and 
other 20 mills prevail as inefficient. We first calculated the projection points to these 20 inefficient mills, and the results are given in Table 2. As we expect and the last row of Table 2 shows, both the total sum of the paper and the BOD are decreased. Also, from Table 2 , we can see that by reducing $8.9 \%$ of the $\mathrm{BOD}, 8.6 \%$ of the paper production is reduced.

Model (8) has been used to calculate the common equilibrium efficient frontier as

$$
F=\left\{(x, y, f): 0.00005 x_{1}+0.00267 x_{2}-0.00461 y_{1}+0.0001 f_{1}+0.01150=0\right\} \cap T_{v}
$$

In model (8), all paper mills are projected onto this frontier, and in this sense all mills are efficient. The new output values are listed in Table 3. An interesting point is that the inputs remain unchanged. The comparison of the fifth column of Table 1 and Table 3 shows that in three mills the BOD values are increased, in two mills they s are decreased and in other 27 mills remain unchanged. Although the summation of BOD is constant. Although the inputs and the total sum of the BOD remain unchanged, but, the total sum of the paper production in all mills are increased from 54.5731 to 211.7466 (the last row of Table 3). This means that to make all mills efficient, we not only need to reallocate the BOD between the mills, but the total output (paper) must also be substantially increased. BOD values for investigated paper mills are shown in Fig. 1.

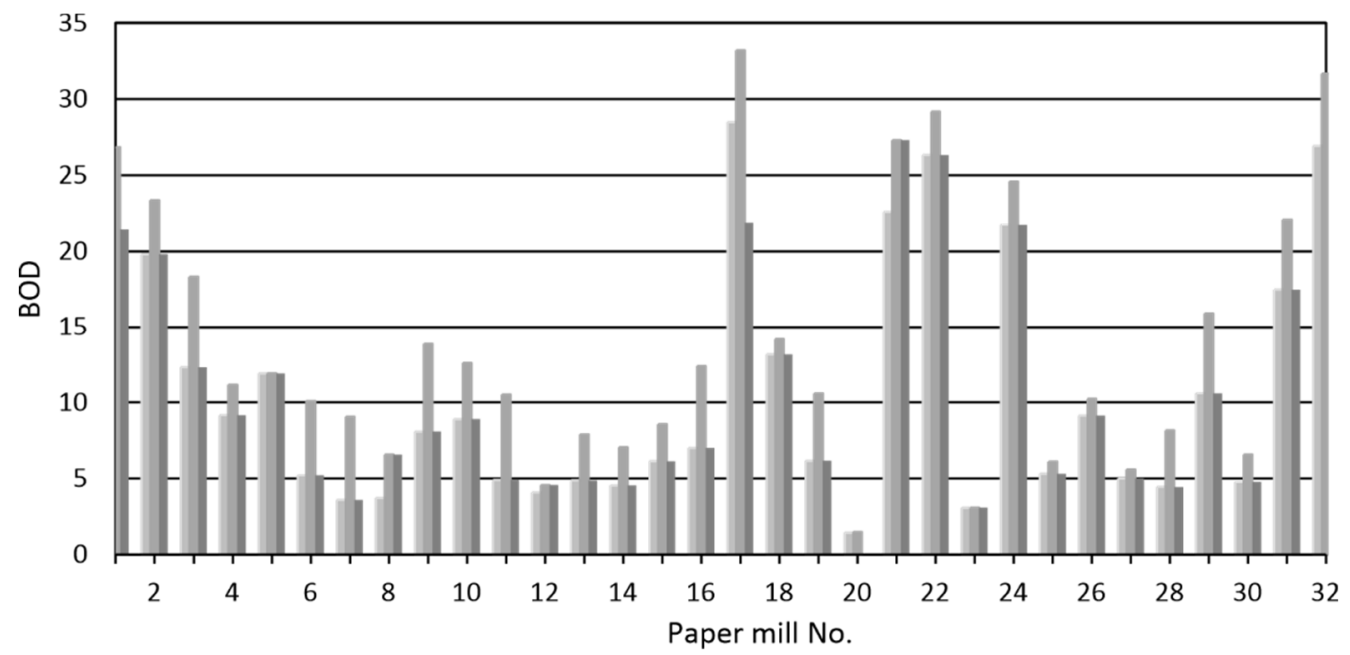

Fig. 1. BOD values for investigated paper mills

As we stated in Section 2, in Wu et al. [17], corresponding to each $D M U$, an ideal competition strategy is defined. This means that the adjusted production possibility set for $D M U_{k}$ is not the same as that for another $D M U$. So, the best practice frontier will 
move if the evaluated $D M U$ changes. However, in our model, a unique common equilibrium efficient frontier is constructed that is not dependent on the unit under evaluation.

Table 3. Results of model (8)

\begin{tabular}{|c|c|c|c|c|c|}
\hline & Paper & Paper- $N$ & BOD-old & BOD-new & BOD-quota \\
\hline 1 & 2.7582 & 11.3625 & 21.4290 & 21.4290 & 26.8452 \\
\hline 2 & 2.9514 & 4.9079 & 19.8062 & 19.8062 & 23.3606 \\
\hline 3 & 1.4700 & 4.6493 & 12.3287 & 12.3287 & 18.3147 \\
\hline 4 & 2.2354 & 12.2052 & 9.1559 & 9.1559 & 11.163 \\
\hline 5 & 2.0669 & 8.9467 & 11.9146 & 11.9146 & 11.9146 \\
\hline 6 & 0.8222 & 9.8808 & 5.2037 & 5.2037 & 10.0819 \\
\hline 7 & 1.5066 & 11.1667 & 3.6054 & 3.6054 & 9.0494 \\
\hline 8 & 0.8066 & 0.8066 & 3.7278 & 6.5775 & 6.5775 \\
\hline 9 & 1.9125 & 2.2183 & 8.0765 & 8.0765 & 13.8485 \\
\hline 10 & 0.7601 & 9.9226 & 8.9060 & 8.9060 & 12.6009 \\
\hline 11 & 1.1579 & 10.8574 & 4.8940 & 4.8940 & 10.5167 \\
\hline 12 & 2.3216 & 2.3216 & 4.0835 & 4.5835 & 4.5835 \\
\hline 13 & 2.1698 & 6.1687 & 4.8750 & 4.8750 & 7.9018 \\
\hline 14 & 0.8127 & 5.9216 & 4.5334 & 4.5334 & 7.0692 \\
\hline 15 & 1.1549 & 5.2370 & 6.1362 & 6.1362 & 8.5654 \\
\hline 16 & 1.0295 & 1.8802 & 7.0186 & 7.0186 & 12.386 \\
\hline 17 & 2.9881 & 8.2966 & 28.4877 & 21.8679 & 33.2039 \\
\hline 18 & 1.9076 & 9.3135 & 13.168 & 13.1680 & 14.168 \\
\hline 19 & 1.2176 & 6.3851 & 6.1616 & 6.1616 & 10.6113 \\
\hline 20 & 0.5187 & 9.5086 & 1.4533 & 0.0000 & 1.5042 \\
\hline 21 & 2.4005 & 2.4005 & 22.5809 & 27.3043 & 27.3043 \\
\hline 22 & 2.3085 & 10.0957 & 26.3390 & 26.3390 & 29.1779 \\
\hline 23 & 0.6545 & 6.4919 & 3.0787 & 3.0787 & 3.0787 \\
\hline 24 & 2.7599 & 6.7999 & 21.7332 & 21.7332 & 24.5954 \\
\hline 25 & 2.3748 & 2.4975 & 5.3061 & 5.3061 & 6.1214 \\
\hline 26 & 1.7323 & 11.3565 & 9.1360 & 9.1360 & 10.2507 \\
\hline 27 & 1.0617 & 5.3416 & 5.0049 & 5.0049 & 5.5956 \\
\hline 28 & 0.9083 & 3.0692 & 4.4373 & 4.4373 & 8.1642 \\
\hline 29 & 1.5151 & 1.8301 & 10.6127 & 10.6127 & 15.9031 \\
\hline 30 & 0.9911 & 8.1034 & 4.7758 & 4.7758 & 6.5614 \\
\hline 31 & 2.5260 & 2.5260 & 17.4677 & 17.4677 & 22.0696 \\
\hline 32 & 2.7721 & 9.2774 & 26.9134 & 26.9134 & 31.6652 \\
\hline Sum & 54.5731 & 211.7466 & 342.3508 & 342.3508 & 444.7538 \\
\hline & & & & & \\
\hline
\end{tabular}

Now, we are interested to know if we use the upper limit of BOD (BOD-quota) as undesirable output, and how this would change the efficiency and paper production. In other words, if we use the BOD quota, do we gain anything substantial? To this end, we solve model (9). The results are listed in Table 4. Nine mills would be evaluated as 
efficient. A $15 \%$ increase in total BOD leads to a $30 \%$ increase in paper production (the last row of Table 4), and a comparison with previous results shows that this does not make sense. Also, by reducing $11.6 \%$ of the BOD-quota, $29.5 \%$ of the paper production is increased.

Table 4. Results of model (9)

\begin{tabular}{|c|c|c|c|c|}
\hline & EFF & Paper & New paper & BOD new \\
\hline 1 & 1.0129 & 2.7582 & 2.7938 & 26.0526 \\
\hline 2 & 1 & 2.9514 & 2.9514 & 19.8062 \\
\hline 3 & 1.8788 & 1.47 & 2.7618 & 18.3147 \\
\hline 4 & 1.1665 & 2.2354 & 2.6077 & 11.163 \\
\hline 5 & 1.2756 & 2.0669 & 2.6365 & 11.9146 \\
\hline 6 & 3.1193 & 0.8222 & 2.5647 & 10.0819 \\
\hline 7 & 1.5731 & 1.5066 & 2.37 & 9.0494 \\
\hline 8 & 1 & 0.8066 & 0.8066 & 3.7278 \\
\hline 9 & 1 & 1.9125 & 1.9125 & 8.0765 \\
\hline 10 & 1.4961 & 0.7601 & 1.1372 & 6.5913 \\
\hline 11 & 2.2284 & 1.1579 & 2.5803 & 10.5167 \\
\hline 12 & 1 & 2.3216 & 2.3216 & 4.0835 \\
\hline 13 & 1.1414 & 2.1698 & 2.4766 & 7.9018 \\
\hline 14 & 1.7593 & 0.8127 & 1.4298 & 7.0692 \\
\hline 15 & 1.687 & 1.1549 & 1.9483 & 8.5654 \\
\hline 16 & 1.9059 & 1.0295 & 1.9621 & 12.386 \\
\hline 17 & 1 & 2.9881 & 2.9881 & 28.4877 \\
\hline 18 & 1.4263 & 1.9076 & 2.7208 & 14.168 \\
\hline 19 & 2.0311 & 1.2176 & 2.473 & 10.6113 \\
\hline 20 & 1.6487 & 0.5187 & 0.8552 & 1.5042 \\
\hline 21 & 1 & 2.4005 & 2.4005 & 22.5809 \\
\hline 22 & 1.2809 & 2.3085 & 2.957 & 21.1322 \\
\hline 23 & 1.627 & 0.6545 & 1.0649 & 3.0787 \\
\hline 24 & 1.0584 & 2.7599 & 2.9211 & 21.0056 \\
\hline 25 & 1.0046 & 2.3748 & 2.3857 & 6.1214 \\
\hline 26 & 1.4841 & 1.7323 & 2.571 & 10.2507 \\
\hline 27 & 1.7898 & 1.0617 & 1.9002 & 5.5956 \\
\hline 28 & 2.0754 & 0.9083 & 1.8851 & 8.1642 \\
\hline 29 & 1.3317 & 1.5151 & 2.0176 & 15.9031 \\
\hline 30 & 1 & 0.9911 & 0.9911 & 4.7758 \\
\hline 31 & 1 & 2.526 & 2.526 & 17.4677 \\
\hline 32 & 1 & 2.7721 & 2.7721 & 26.9134 \\
\hline Sum & 46.0023 & 54.5731 & 70.6903 & 393.0611 \\
\hline & & & & \\
\hline
\end{tabular}

The results of the first and second alternatives of Wu et al. [16], along with the results of our proposed model, are given in Table 5 (the results are given in total sense). As the last row of Table 5 shows, the new inputs remain unchanged in our approach. By 
fixing the total BOD production, the paper production is increased to 211.7466. However, the results show that in Wu et al. [16], the second input, capital, must be increased in both alternatives. In response to this change in inputs, the paper production is almost double in the first alternative, and it remains unchanged in the second alternative. All methods made all mills efficient.

Table 5. Summary of the results

\begin{tabular}{|l|c|c|c|c|}
\hline \multicolumn{1}{|c|}{ Source } & Labor & Capital & Paper & BOD \\
\hline Original values & 20158 & 113.0903 & 54.5731 & 342.3508 \\
\hline The first alternative of Wu et al. [16] & 20158 & 150 & 111.1425 & 350.8636 \\
\hline The second alternative of Wu et al. [16] & 20158 & 147.1853 & 54.5731 & 111.2030 \\
\hline The proposed approach & 20158 & 113.0903 & 211.7466 & 342.3508 \\
\hline
\end{tabular}

\section{Conclusions}

In real applications of DEA, there are production processes in which undesirable products are produced along with the desirable outputs, and due to some limitations the total sum of undesirable outputs are fixed and the decision-maker has no control over reducing these products in the total sense. However, the trade-off between the DMUs is possible. The problem of modelling fixed-sum undesirable outputs in production processes is studied in this paper. In most studies on fixed-sum undesirable output, all $D M U$ s make the least adjustment to construct a new equilibrium efficient frontier. So, different $D M U$ s are evaluated, based on the different efficient frontiers. This study proposes a DEA-based equilibrium efficient frontier in which the $D M U$ s with fixed-sum outputs are evaluated fairly.

Taking the weak disposability assumption of Shephard [15] into consideration, and using a directional distance function, a linear programming problem is proposed to construct a common equilibrium efficient frontier. Moreover, to compare the proposed approach with other approaches, we use a real case of 32 paper mills in Anhui province, China. In this case, the BOD, as an undesirable output, is considered as fixed-sum output and, after reallocating, all provinces have become as efficient.

The advantage of the proposed approach is that it provides a single equilibrium efficient frontier, and all $D M U$ s are projected onto this frontier. In this sense, the computational effort is relatively low.

A drawback of constructing a single equilibrium efficient frontier is that in some real cases, we need a large trade-off between the $D M U$ s, and this may be impossible or infeasible in practical applications. To overcome this, we suggest using outliers in DEA. This could be our future research. 


\section{References}

[1] Amirteimoori A., FouladVAND M., Kordrostami S., Efficiency measurement using nonparametric production analysis in the presence of undesirable outputs. Application to power plants, Oper. Res. Dec., 2017, 27 (3), 5-20.

[2] Amirteimoori A., Masrouri S., YANG F., Kordrostami S., Context-based competition strategy and performance analysis with fixed-sum outputs. An application to banking sector, Oper. Res. Soc., 2017, 68, 1461-1469.

[3] BANKer R.D., ChARNeS A., COOPER W.W., Some models for estimating technical and scale inefficiencies in data envelopment analysis, Manage. Sci., 1984, 30, 1078-1092.

[4] Charnes A., CoOper W.W., Rhodes E., Measuring the efficiency of decision-making units, Eur. J. Oper. Res., 1978, 2 (6), 429-444.

[5] FÄre R., Grosskopf S., Non-parametric productivity analysis with undesirable outputs. Comment, Amer. J. Agr. Econ., 2003, 85, 1070-1074.

[6] GoMES E.G., LiNs M.P.E., Modelling undesirable outputs with zero sum gains data envelopment analysis models, Oper. Res. Soc., 2008, 59 (5), 616-623.

[7] Hailu A., VeEman T.S., Non-parametric productivity analysis with undesirable outputs. An application to the Canadian pulp and paper industry, Am. J. Agr. Econ., 2001, 83, 605-616.

[8] Khoshandam L., Kazemi Matin R., AmirTeimoori A., Marginal rates of substitution in data envelopment analysis with undesirable outputs. A directional approach, Measurement, 2015, 68, 49-57.

[9] KUOSMANEN T., Weak disposability in nonparametric production analysis with undesirable outputs, Am. J. Agr. Econ., 2005, 87, 1077-1082.

[10] Lins M.P.E., Gomes E.G., Soares De Mello J.C.C.B., SoAres De Mello A.J.R., Olympic ranking based on a zero sum gains DEA model, Eur. J. Oper. Res., 2003, 148, 312-322.

[11] Momeni E., Hosseinzadeh Lotfi F., Farzipoor SAen R., NAJAfi E., Centralized DEA-based reallocation of emission permits under cap and trade regulation, J. Clean. Prod., 2019, 234, 306-314.

[12] Pham M.D., Zelenyuk V., Weak disposability in nonparametric production analysis. A new taxonomy of reference technology sets, Eur. J. Oper. Res., 2019, 274 (1), 186-198.

[13] Sadri S., Rostamy-Malkhalifeh M., Shoja N., A new method for optimization of inefficient cost units in the presence of undesirable outputs, Int. J. Ind. Math., 2018, 10 (4), 331-338.

[14] SEIFORD L., ZHU J., Modeling undesirable factors in efficiency evaluation, Eur. J. Oper. Res., 2002, $42,16-20$.

[15] ShePhaRd R.W., Theory of cost and production functions, Princeton University Press, Princeton 1970.

[16] Wu J., An Q., Ali S., LIANg L., DEA based resource allocation considering environmental factors, Math. Comp. Model., 2013, 58, 1128-1137.

[17] Wu J., AN Q., YaO X., WANG B., Environmental efficiency evaluation of industry in China based on a new fixed sum undesirable output data envelopment analysis, J. Clean. Prod., 2014, 71, 96-104.

[18] YAng F., Wu D.D., LiAng L., O'NeILL L., Competition strategy and efficiency evaluation for decision-making units with fixed-sum outputs, Eur. J. Oper. Res., 2011, 212, 560-569.

[19] Y ANG M., Li Y., CHEN Y., Liang L., An equilibrium efficiency frontier data envelopment analysis approach for evaluating decision-making units with fixed-sum outputs, Eur. J. Oper. Res., 2014, 239, 479-489.

[20] YANG M., Li Y., LiANG L., A generalized equilibrium efficient frontier data envelopment analysis approach for evaluating DMUs with fixed-sum outputs, Eur. J. Oper. Res., 2015, 246 (1), 209-216.

[21] Zare-Haghighi H., Rostamy-Malkhalifeh M., Jahanshahloo M., Measurement of congestion in the simultaneous presence of desirable and undesirable outputs, J. Appl. Math., 2014, SI22, 1-9.

[22] Zhang J., Wu Q., Zhou Z., A two-stage DEA model for resource allocation in industrial pollution treatment and its application in China, J. Clean. Prod., 2019, 228, 29-39. 